\title{
Iliopsoas Hematomas in a Patient with Progressive Encephalomyelitis with Rigidity and Myoclonus
}

\author{
Rui Shimazaki, Masako Mukai, Utako Nagaoka, Keizo Sugaya and Kazushi Takahashi
}

\begin{abstract}
:
Progressive encephalomyelitis with rigidity and myoclonus (PERM) is a rare and severe syndrome characterized by rigidity of the limb and truncal muscles, brainstem signs, myoclonus, and hyperekplexia. Iliopsoas hematoma is a serious complication of bleeding disorders that occurs most commonly in patients with hemophilia and also in association with anti-coagulant drug treatment. We herein present a case of PERM complicated with bilateral iliopsoas hematomas. His neurological symptoms improved after immunotherapy, and thereafter the iliopsoas hematomas disappeared. Neurologists should consider iliopsoas hematomas as a serious potential complication of PERM.
\end{abstract}

Key words: PERM, progressive encephalomyelitis with rigidity and myoclonus, iliopsoas hematomas, glycine receptor antibodies, hypercontraction

(Intern Med 60: 2475-2477, 2021)

(DOI: 10.2169/internalmedicine.6604-20)

\section{Introduction}

Progressive encephalomyelitis with rigidity and myoclonus (PERM) is a rare and severe syndrome characterized by rigidity of the limb and truncal muscles, brainstem sign, myoclonic jerk, autonomic dysfunction, cognitive impairment and hyperekplekia $(1,2)$. Glycine receptor autoantibodies have been found in PERM patients and they are thought to be the autoantibodies responsible for the pathology of this disorder (3). Iliopsoas hematoma is a rare and life-threatening complication of bleeding disorders that occurs in patients with clotting disorders and also in association with anti-coagulant drug treatments (4-6). It is the most frequent iliopsoas hematoma in anticoagulation-related conditions, and it causes abdominal and lumbar pain, anemia, muscle dysfunction and occasionally femoral nerve palsy (7). We herein report the first case of PERM complicated with bilateral iliopsoas hematomas.

\section{Case Report}

A 63-year-old Japanese man presented with a 7-month history of bilateral painful lower limb muscle stiffness, diffi- culty in walking and dysphagia. He had no history of bleeding disorders or anticoagulation therapy and was admitted to a local hospital. On laboratory testing, mild anemia was detected (hemoglobin; $10.1 \mathrm{~g} / \mathrm{L}$, normal range: 12.0-16.0 g/L). Factor XIII (FXIII) activity was reduced to $16 \%$ (normal range: $70-140 \%$ ). Other data related to coagulation showed normal ranges, including platelet count, Factor II, Factor V, Factor VII, Factor VIII, Factor IX and Factor X. A body CT scan showed bilateral swelling of the iliopsoas muscles, right adductor muscle, and left external obturator muscle (Fig. 1A, D). The diagnosis of iliopsoas hematoma was made by percutaneous CT-guided fine-needle aspiration of the muscle, in which bloody fluid without bacterial contamination was detected. A malignancy survey showed no remarkable findings. The patient's neurological symptoms had gradually worsened. Eight months after disease onset, he was transferred to our hospital. On admission, he was slightly somnolent. A neurological examination revealed persistent horizontal gaze-evoked nystagmus, downbeat nystagmus, lower hemifacial stiffness on the right side, bulbar palsy, a limitation of tongue movement, and lock jaw due sustained muscle contraction. His extremities were rigid, and it was difficult to bend his knee joints even passively. He also showed intermittent hiccups and paroxysmal myoclonic 

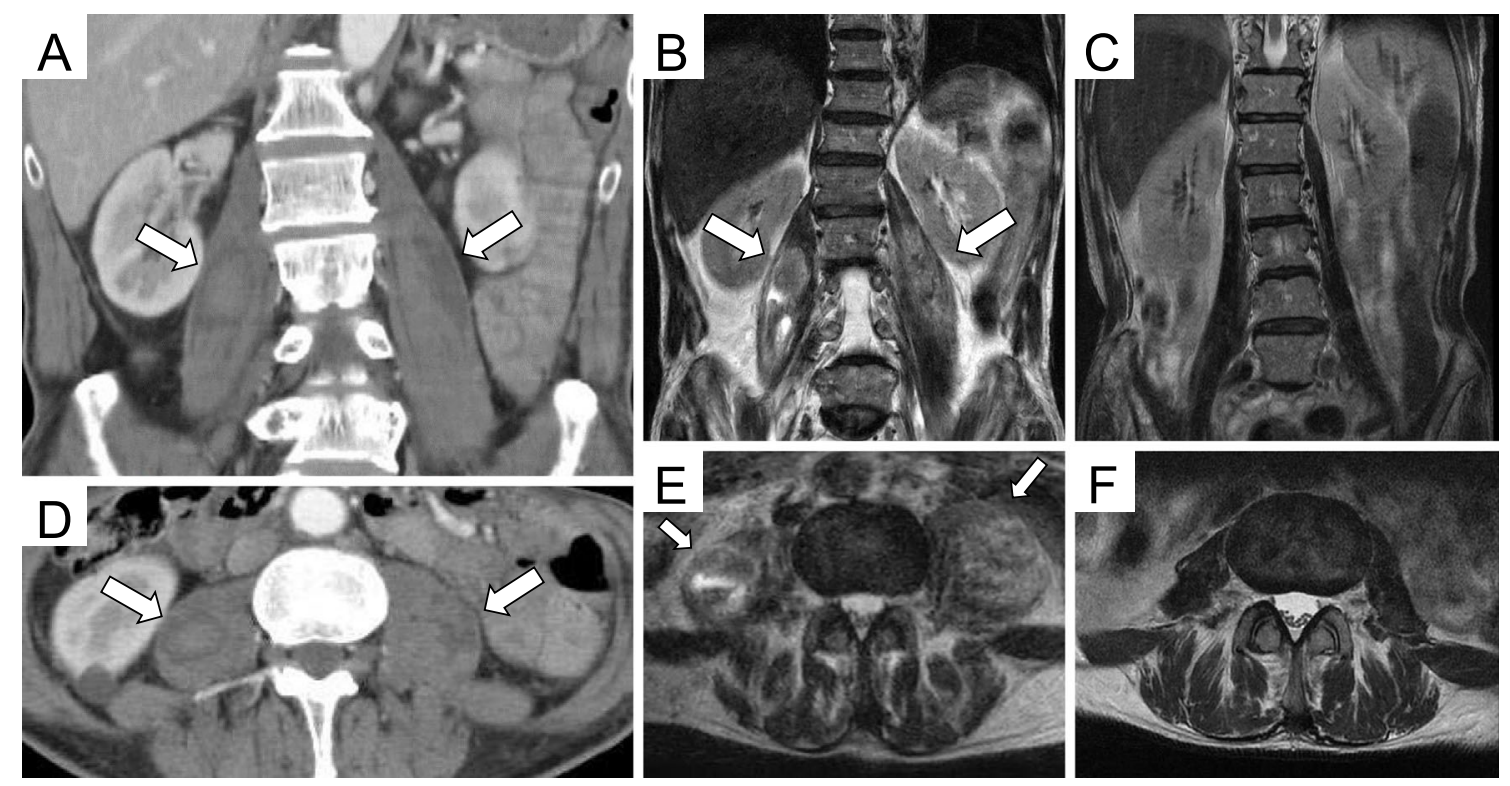

Figure 1. Contrast-enhanced CT and MRI findings on admission day 18 (A, B, D, E) and on admission day 65 (C, F). A coronal view of contrast-enhanced CT and MRI T2 weighted image (A, B, C). An axial view of contrast-enhanced $\mathrm{CT}$ and MRI T2 weighted image (D, E, F). Before treatment, contrast-enhanced CT showed enlarged muscle with enhanced effect (A, D; arrows). MRI showed bilateral iliopsoas hematomas (B, E; arrows). After treatment, the bilateral iliopsoas hematomas had completely disappeared $(\mathrm{C}, \mathrm{F})$.

jerks in his back and lower limbs, which were easily provoked by sudden noise, bright light, and tactile stimuli. Laboratory tests showed mild anemia (hemoglobin; $10.9 \mathrm{~g} /$ L). He tested negative for anti-glutamic acid decarboxylase. Magnetic Resonance Imaging (MRI) showed bilateral iliopsoas hematomas (Fig. 1B, E). Electromyography of the patient's lower limb muscles showed small polyphasic motor unit action potentials with early recruitment. A nerve conduction study showed no remarkable findings. A surface electromyogram examination showed simultaneous continuous motor unit activity in the tibialis anterior and gastrocnemius. A diagnosis of PERM was considered, and glycine receptor (GlyR) antibodies were found to be positive in his serum and cerebrospinal fluid. GlyR autoantibodies have been found in PERM patients and are thought to be the autoantibodies responsible for the pathology of this disorder (3). After aggressive immunotherapy with 2 cycles of intravenous immunoglobulin therapy $(400 \mathrm{mg} / \mathrm{kg} / \mathrm{day}$ for 5 days $)$ and 2 cycles of intravenous methylprednisolone $(1,000 \mathrm{mg}$ per day for three days), followed by the oral intake of prednisolone (60 mg/day or $1.5 \mathrm{mg} / \mathrm{kg}$ of body weight/per day), hyperekplexia, myoclonic jerks, and stiffness of extremities were alleviated, and his FXIII activity had returned to a nearnormal level (65\%) (Fig. 2). A follow-up MRI revealed the disappearance of bilateral iliopsoas hematomas (Fig. 1C, F). He thereafter could walk with assistance and then finally consume a normal diet.

\section{Discussion}

GlyRs are widely present in the spinal cord and brain- stem (8). Autoantibodies against GlyR were identified in patients with PERM and were found to disrupt glycinergic neurotransmission (3). A condition similar to PERM is tetanus, in which a disruption of the physiologic inhibition of alpha motor neurons leads to muscle hypercontraction and hyperekplexia. Tetanus is caused by the tetanus toxin-the product of Clostridium tetani-which inhibits the release of GABA and glycine (9). There have been reports describing intramuscular hematomas in patients with tetanus (10). The incidence of tetanus-related iliopsoas hematomas was higher than that of anticoagulation-related iliopsoas hematomas, suggesting that the muscular injury induced by muscle spasms might be the underlying cause of iliopsoas hematomas in tetanus patients (10). A simple microtrauma due to isometric muscle contraction could potentially result in muscle and capillary tears, subsequently leading to spontaneous muscle hematomas (11). Thus, PERM itself may confer a risk of developing intramuscular hematoma, although as far as we could determine, no other case of PERM-complicated intramuscular hematoma has been reported to date. Laboratory tests showed a low activity of FXIII. A plasma FXIII activity as low as 5\% was previously considered sufficient for normal hemostasis (12). However, the FXIII level used to delineate the boundary between hemostasis and hemorrhage could be much higher. A low FXIII activity is caused by the inhibition of FXIII or decreased synthesis and/or an increased consumption of FXIII and is often observed in patients with enlarged intramuscular hematoma (13). These findings, together with the negative result for anti-FXIII autoantibodies, suggest that the low FXIII activity in our patient reflected an excessive consumption of FXIII due to 


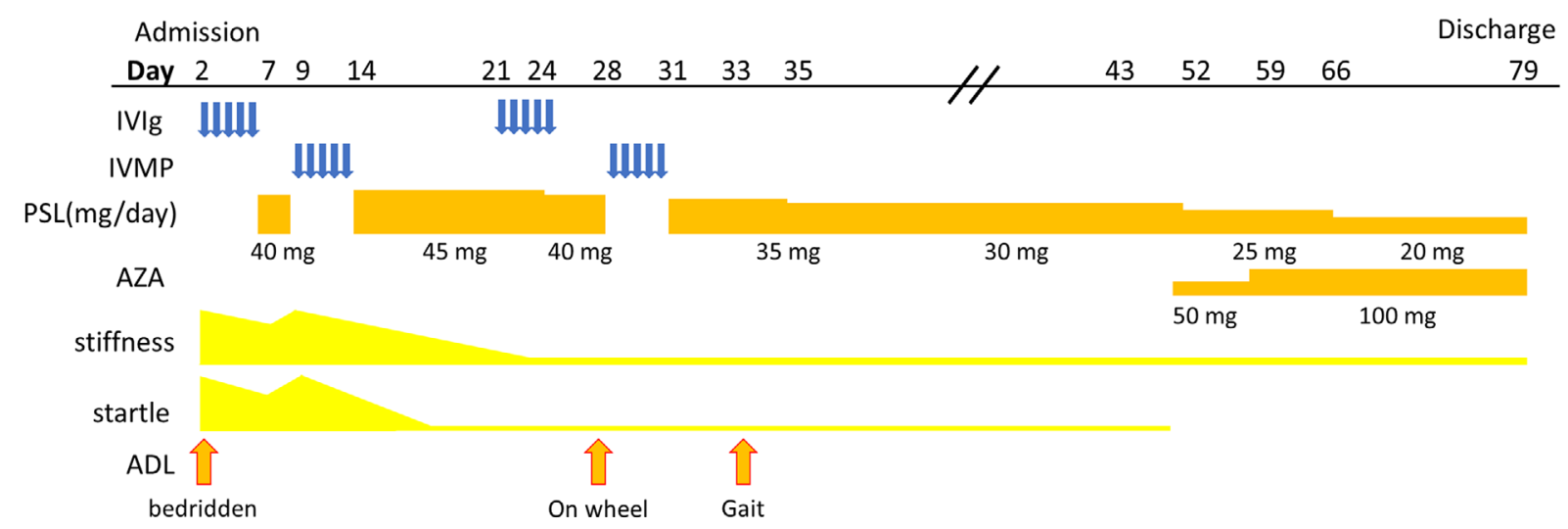

Figure 2. The time course of hospitalization. IVIG: intravenous immunoglobulin, IVMP: intravenous methylprednisolone, PSL: prednisolone, AZA: azathioprine

iliopsoas hematomas. However, we cannot dismiss the possibility that the low FXIII activity may have played a role in the formation of iliopsoas hematomas. Iliopsoas hematomas cause a sudden deterioration of the patient's clinical condition and they can become life-threatening (11), but their early detection seems to be difficult in PERM patients because PERM patients tend to have painful lower limb muscle spasms. Therefore, neurologists should actively survey for intramuscular hematoma, including iliopsoas hematoma when progressive anemia is present in PERM patients. MRI is a sensitive option for diagnosing intramusclar hematomas, and CT is also useful for diagnosing intramusclar hematomas (14).

The authors state that they have no Conflict of Interest (COI).

\section{References}

1. Hutchinson M, Waters P, McHugh J, et al. Progressive encephalomyelitis, rigidity, and myoclonus: a novel glycine receptor antibody. Neurology 71: 1291-1292, 2008.

2. Stern WM, Howard R, Chalmers RM, et al. Glycine receptor antibody mediated progressive encephalomyelitis with rigidity and myoclonus (PERM): a rare but treatable neurological syndrome. Pract Neurol 14: 123-127, 2014.

3. Crisp SJ, Dixon CL, Jacobson L, et al. Glycine receptor autoantibodies disrupt inhibitory neurotransmission. Brain 142: 3398-3410, 2019.

4. Yogarajah M, Sivasambu B, Jaffe EA. Spontaneous iliopsoas haematoma: a complication of hypertensive urgency. BMJ Case Rep 2015: bcr2014207517-b, 2015.
5. Kheiri B, Al Salihi, Maldonado D, Nakhleh R, Bachuwa G. Warfarin-induced spontaneous iliopsoas hematoma - an unusual complication. Clin Case Rep 6: 1639-1640, 2018.

6. Balkan C, Kavakli K, Karapinar D. Iliopsoas haemorrhage in patients with haemophilia: results from one centre. Haemophilia 11: 463-467, 2005.

7. Saad Z, Ahmed B, Mostafa R, Hicham B, Lahcen B. Conservative treatment of a psoas hematoma revealed by a lower limb palsy. Pan Afr Med J 28: 2017.

8. Baer K, Waldvogel HJ, Faull RL, Rees MI. Localization of glycine receptors in the human forebrain, brainstem, and cervical spinal cord: an immunohistochemical review. Front Mol Neurosci 2: $25,2009$.

9. Hassel B. Tetanus: pathophysiology, treatment, and the possibility of using botulinum toxin against tetanus-induced rigidity and spasms. Toxins 5: 73-83, 2013.

10. Ishii N, Mochizuki H, Shiomi K, Nakazato M. Iliopsoas hematoma as a complication of tetanus. Neurol Clin Neurosci 7: 26-30, 2019.

11. Dohan A, Darnige L, Sapoval M, Pellerin O. Spontaneous soft tissue hematomas. Diagn Interv Imaging 96: 789-796, 2015.

12. Ichinose A. Hemorrhagic acquired factor XIII (13) deficiency and acquired hemorrhaphilia 13 revisited. Semin Thromb Hemost 37: 382-388, 2011.

13. Saotome K, Koguchi Y, Tamai K, Sakai H, Ohno W, Yamato M. Enlarging intramuscular hematoma and fibrinolytic parameters. J Orthop Sci 8: 132-136, 2003.

14. Seo JG, Yang JC, Kim TW, Park KH. Intramuscular hematoma on the psoas muscle. Korean J Neurotrauma 15: 234, 2019.

The Internal Medicine is an Open Access journal distributed under the Creative Commons Attribution-NonCommercial-NoDerivatives 4.0 International License. To view the details of this license, please visit (https://creativecommons.org/licenses/ by-nc-nd/4.0/).

(C) 2021 The Japanese Society of Internal Medicine Intern Med 60: 2475-2477, 2021 